\title{
Fish'n'Steps: Encouraging Physical Activity with an Interactive Computer Game
}

\author{
James J. Lin, Lena Mamykina, Silvia Lindtner, Gregory Delajoux, \\ and Henry B. Strub \\ Siemens Corporate Research, \\ 755 College Road East, Princeton, NJ 08540 USA \\ \{jameslin, lena.mamykina, silvia.lindtner.ext, hank.strub\} \\ asiemens.com, gregory.delajoux@gmail.com \\ http://www.scr.siemens.com
}

\begin{abstract}
A sedentary lifestyle is a contributing factor to chronic diseases, and it is often correlated with obesity. To promote an increase in physical activity, we created a social computer game, Fish'n'Steps, which links a player's daily foot step count to the growth and activity of an animated virtual character, a fish in a fish tank. As further encouragement, some of the players' fish tanks included other players' fish, thereby creating an environment of both cooperation and competition. In a fourteen-week study with nineteen participants, the game served as a catalyst for promoting exercise and for improving game players' attitudes towards physical activity. Furthermore, although most player's enthusiasm in the game decreased after the game's first two weeks, analyzing the results using Prochaska's Transtheoretical Model of Behavioral Change suggests that individuals had, by that time, established new routines that led to healthier patterns of physical activity in their daily lives. Lessons learned from this study underscore the value of such games to encourage rather than provide negative reinforcement, especially when individuals are not meeting their own expectations, to foster long-term behavioral change.
\end{abstract}

\section{Introduction}

In recent decades, obesity has become a problem on the scale of a world-wide epidemic. The 1999 National Health and Nutrition survey (NHANES) estimated that $61 \%$ of US adults are either overweight or obese. These people suffer from both deleterious health consequences and the corresponding psychological stigma [1] . Epidemiologic studies have identified several environmental factors that contribute to this continual gain of weight over recent decades. Lifestyles have become increasingly sedentary (e.g. less physical activity, commonly combined with more time spent watching television) and energy-dense foods (high-fat, concentrated-sugar, low-fiber) have become the common components of individuals' diets [2] .

The most effective approaches to treating people for being overweight or obese are similar to those for other chronic diseases. They begin with lifestyle improvements, and continue to more invasive treatments such as pharmaceuticals and even surgery. 
Lifestyle improvements to control weight include exercise, diets that restrict calories, fat or carbohydrates, or a combination of both exercise and diets. Unfortunately, there is limited evidence of successful weight management through lifestyle alterations. "Studies paint a grim picture: those who complete weight-loss programs lose approximately $10 \%$ of their body weight, only to regain two-thirds of it back within a year and almost all of it back within 5 years" [3] . One of the main reasons for such a high relapse rate is that individuals have difficulty maintaining the necessary lifestyle improvements.

Many factors contribute to the challenges of maintaining lifestyle improvements. Some are economic, such as the availability and cost of healthy foods or the cost of joining a health club and the time required to regularly exercise. Others are psychological, for example depression and a lack of motivation to follow a healthy lifestyle every day when the improvements are only slowly noticeable. Even others are social, including the desire to fit in with one's existing social circles who engage in their own unhealthy behaviors [1] [4] . However, the desired changes can be achieved without a significant disruption of one's current lifestyle. For example, studies have demonstrated that individuals who walked at least 10,000 steps a day were more likely to maintain their desired weight [5] and [24] .

Individual behavior change has been a subject of active investigation in the areas of cognitive and clinical psychology. One of the most widely accepted theoretical models is the Transtheoretical Model (TTM), introduced by James Prochaska [6] . TTM argues that individuals change their behavior gradually, by advancing along a series of steps. These steps vary from pre-contemplation in which individuals have not realized the need for change, to termination in which the new behavior has become so habitual that there is no longer any danger of relapse. TTM has been successfully used to design interventions for such undesirable behaviors as alcoholism, smoking and domestic violence [6] [7] [8] and to motivate increase in exercise and other types of physical activity [9] [10] [11] . However, traditional techniques inspired by TTM rely on significant clinical resources for the individuals who seek the change. There is a need to develop more innovative and cost-effective intervention programs that supplement or replace meetings with a therapist [11] .

In the recent years a number of innovative programs introduced novel technologies to reduce the cost of continuous involvement of clinical personnel. Many of these techniques transform physical exercise into engaging individual or social games that often mix real and virtual environments [12] [13] [14] . In an alternative approach, pedometers, small electronic devices that monitor individual step counts have been used as a ubiquitous and unobtrusive motivational technique available anytime and anywhere [5] [15] [16] .

In this paper we describe Fish'n'Steps, an application that combines ubiquity and simplicity of pedometers with the engagement of social computing games (Fig. 1). Individuals enrolled in the game were provided with pedometers to measure their daily step count. The number of steps taken each day was then mapped to the growth and emotional state of a virtual pet that was "given" to each individual: a fish in a fish-tank. Additional incentives were designed to incorporate social dynamics, such as competition between teams of players. 

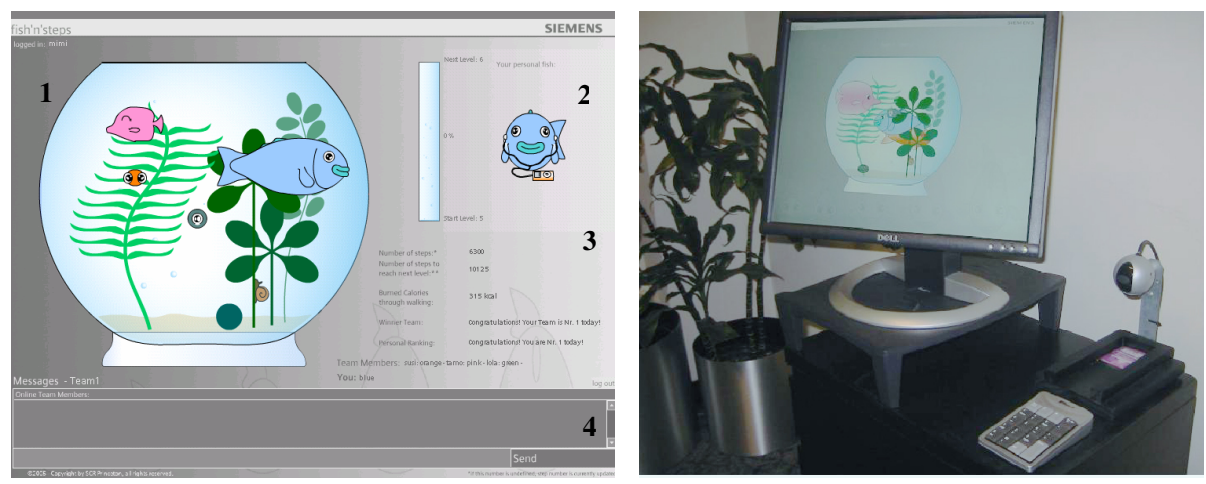

Fig. 1. One participant's display after approximately two weeks into the trial in the Fish'n'Steps team-condition, also the public kiosk and pedometer platform, which rotated through each of the team fish-tanks. The components of the personal display include: 1) Fish Tank - The fish tank contains the virtual pets belong to the participant and his/her team members, 2) Virtual Pet - The participant's own fish in a frontal view on the right side next to the fish tank, 3) Calculations and feedback - improvement, burned calories, progress bar, personal and team ranking, etc., 4) Chat window for communicating with team members.

To evaluate the effect of Fish'n'Steps, we recruited 19 participants from the staff of Siemens Corporate Research to participate in a 14-week study. Two experimental conditions were designed to separately assess the impact of the virtual pet and the social influences. Application of the TTM to assess behavior that changed during the study demonstrated that Fish'n'Steps was a catalyst of a positive change for 14 out of 19 participants. This effect was evident in either an increase in their daily step count (for 4 participants), a change in their attitudes towards physical activity (for 3 participants) or a combination of the two (for 7 participants). The greatest change in daily number of steps was by the participants who were at the TTM's intermediate levels of the behavior change. For these participants the game provided just enough motivation to translate mental readiness into action.

While the overall findings were encouraging, there are a number of possibilities for future investigations. For example, selecting participants from our own research organization limited representativeness of the sample to highly educated individuals relatively open to adopting new technologies. In addition, the game highlighted the importance of careful selection of incentives: unachievable or not challenging goals can fail to inspire the desired change.

\section{Interventions for Behavior Change}

There are a variety of techniques developed over the years to motivate behavior change. Traditional techniques are usually delivered by a trained specialist in either individual or group settings. Examples of these techniques include goal-setting, selfassessment, or monitoring of achieved progress [17] . Two particular approaches that influenced the current project include motivating behavior change by cultivating a 
strong internal locus of control through care of pets or plants [18] [19] and incorporating social influences through family participation [20] . For example, in a classical study of elderly people living in an assistive care facility, such simple interventions as an opportunity to choose and then care for a plant led to a significant increase in the sense of control over one's life and improvement in health [18]. At the same time, engaging family members in a cooperative weight monitoring effort has been shown to lead to more enduring effects than those achieved through an individual's own motivation [20] .

New techniques developed in the recent years complement and at times replace the traditional physician involvement by introducing advanced computing technologies. These computerized interventions have a number of advantages. In addition to being cost-efficient, they can be delivered anytime and anywhere, or "just in time" for a decision - when they can have the highest potential impact. In studies conducted in public places, motivational messages delivered at the time of decision making inspired individuals to take simple steps to improving their health, such as choosing a staircase over the escalator [21]. At the same time, sensor technologies can be used to make these messages highly personalized. For example, artistic sculptures resembling human figures were used to enhance individuals' awareness of their posture [22] . Often, these techniques incorporate the emotional aspects, or persuasive computing techniques [23] . For example, an attachment to virtual creatures, such as Tamagotchi, inspired technological health interventions for children with chronic diseases [19] . In these games, strong and attractive animated characters have the same diseases as children who play the game, for example asthma or diabetes, thus providing positive role-models and reducing the stigma associated with the child's disease.

There are a number of applications that motivate increase in physical activity in a fun way through engaging individuals in games that mix real and computing worlds. These games became known as Serious Games or Exergames. For example, "Tagaboo", a collaborative children's game, focuses on developing coordination through playful interaction [24] . Exercise Bicycles enriches the experience of an indoor exercise bicycle in engaging users in a Virtual Environment single-player game [13] .Yet another, and perhaps the most researched Serious Game, Dance Dance Revolution connects a sensor-enabled dance floor with a video interface and provides stimulating exercise as a social activity - dance competition [12] .

An alternative approach to motivating physical activity that became popular in the resent years utilizes pedometers, small battery operated devices that count how many steps individuals' take each day [5] [15] . Usage of pedometers helps individuals realize their current level of activity, set achievable goals and monitor their progress towards the goals, either individually or in a social setting. For example, in a study by Consolvo et al [16], groups of friends wearing pedometers could share each others' goals and progress via mobile phones. In addition, devices like Body-Bugg by BodyMedia can provide such measurements as consumed or burned calories.

While challenging and exciting, games like Tagaboo, Dance Dance Revolution and the Exercise Bicycle require focused engagement and are constrained in time and location. In our work, we looked for less focused and more ubiquitous ways to promote physical activity. At the same time, utilizing pedometers achieves the 
desired ubiquity but could benefit from providing additional motivation and incentives. Fish'n'Steps, described in this paper attempts to combine both, ubiquity and unobtrusiveness of pedometers, with engaging gaming approach of Serious Games.

\section{Fish'n'Steps}

\subsection{Step Capture}

"Fish'n'Steps" was built as a distributed software application that included several functioning components as well as some "Wizard of Oz" components. Simple commercially available pedometers, Sportline 330, were used to measure the step count of individual participants. The main considerations for selection of this model were its low price, large display with overall small size, and the cumulative step count display, rather than daily step count with overnight reset, which simplified the automated upload. However, the cumulative step count display, while increasing the value of the interventions in the game, reduced the overall benefit of the pedometers, To collect data from pedometers, individuals placed their pedometer on a platform at a public kiosk (Fig. 1), and took a picture of their pedometer screen, including the unique pedometer ID. The picture was captured and sent to a member of the research team who entered the appropriate data into a database. Due to the competitive nature of the game, such automated upload was essential to prevent tampering with the game and adjusting numbers to help one's team win. This daily upload could neither measure nor prevent tampering through shaking the pedometer while sitting to increase step count or giving the pedometer to somebody else to wear. While it was hard to avoid these more involved types of tampering, automated upload at least prevented false reports. In addition, kiosk upload combined with a public display was meant to simplify the upload procedures, and to foster individuals' interest in the game.

\subsection{Establishing Daily Goals}

Once an individual's daily step number was uploaded into the database, the application calculated the incremental daily increase and compared it with a personal goal that had been established for each individual during the pre-study phase. After reviewing previous pedometer studies [5] [15] , 3 heuristics were used to calculate custom goals for each participant:

Each participant should have a reasonable target over the 6 week intervention period. In previous studies, the average increase in step count was 2-3000 steps [15] [25] ;

The increase would be negatively correlated with participants' baseline step count; An upper bound was set at 12,000 steps per day, since participants in previous studies tended to reach a plateau of 10-12,000 steps.

For example, a participant with a baseline of 3000 daily steps had an overall goal of 5250 steps, while a participant with a baseline of 11,000 daily steps had an overall goal of 11,630 steps. Although these goals may not seem ambitious, the study's incentive structure depended on participants receiving positive feedback to help guide them through daily encouragement. 
These specific 6-week goals were calculated using an exponential function to set a goal for each week. Daily progress was sufficient when individuals reached their daily goal (1/5 of their weekly goal), nearly sufficient when they reached half of their daily goal, and insufficient when they did not reach even half of their daily goal. Subjects whose improvement exceeded their weekly goal were given a new goal based on an adjustment of their baseline.

\subsection{Developing Incentives}

Individuals' daily progress towards their goals was mapped to the development of the fish in two different ways. The daily step count for each participant contributed to the growth of his or her fish in a step-wise fashion. As soon as the total number of steps exceeded a predefined target, the fish's appearance changed to the next growth level (Fig. 2). If the fish's upper appearance level was reached, an additional ("baby") fish was attached to the fish tank, which grew according to the further increase of the user's step count. At the same time, success in reaching a participant's daily goal affected the facial expression of his or her fish-making it happy (in case of sufficient progress), angry (in case of nearly sufficient progress), or sad (insufficient progress) (Fig. 2).
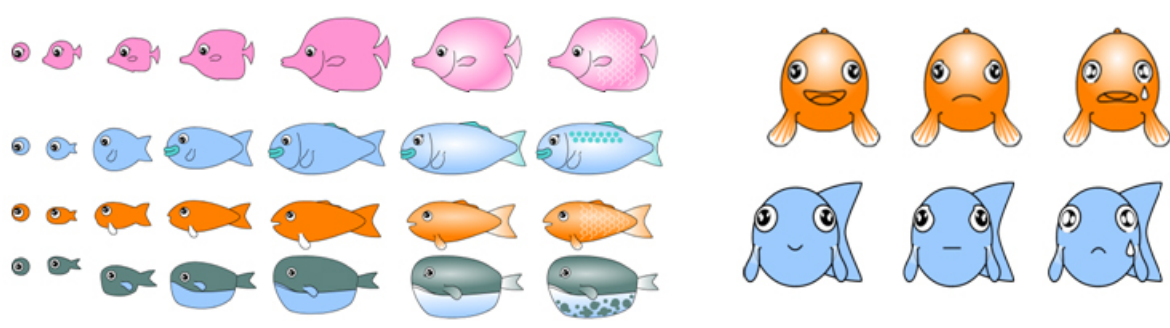

Fig. 2. Seven growth levels (left panel) and examples of three types of facial expressions (right panel) of the virtual characters

Two different versions of the game interface were used to create two experimental conditions. In one version, each participant's fish had its own fish tank and hence was not involved in a competition process. In the second version, each fish-tank was shared by four participants' fish. In this condition, the progress of each fish affected the entire fish tank: each "insufficient" day for any team member resulted in the tank's water getting darker, as well as the gradual removal of the fish-tank's decorations (plants, small animals, such as snails, etc.). Individuals within teams saw each others' progress, and could converse over an anonymous chat application.

Two displays were designed for the application. The public display was placed next to the upload kiosk and rotated through each group's fish-tank (Fig. 1), recognizable through the team's number. The public display was designed to promote a daily competition between teams for the "healthiest" fish tank. At the same time, individuals of both conditions could $\log$ in on their own computer to monitor the progress of their own fish, as well as other fish in their group's fish-tank. 
The game application was realized as a web interface created with Macromedia Flash. The Flash Interface combined the game logic with input from a mySql database (daily number of steps, group and individual ranking, user properties). PHP language was used for communication between the Flash client and the Apache server.

\section{Study Description}

\subsection{Participants}

To assess the effectiveness of Fish'n'Steps, 19 participants (11 females and 8 males, aged from 23 to 63) were recruited among the staff of Siemens Corporate Research, Inc, The participants of the study were a relatively homogeneous group in terms of education (graduate degree), working environment, and even living environments (suburban New Jersey). However, as is discussed below, there were fairly large individual variations in their lifestyles and attitudes towards physical exercise. These span from individuals never having, nor wishing for an exercise routine, to individuals who exercised rigorously and regularly. The average daily step count collected during the pre-study reflected these differences, with numbers ranging from 3,700 to over 11,000 . The participants were compensated $\$ 25$ for enrolling in the 14 weeks study.

The selection of participants in this study received a lot of consideration. The research team was looking for participants with sedentary jobs that did not require physical activity. However, the need for automated step capture suggested recruitment of co-located participants. At the time of the study, the only pedometer that allowed upload to a PC via serial cable was not available in the US market. As a result, we developed an upload kiosk and invited fellow employees from Siemens Corporate Research (SCR) to volunteer for the study. This decision had limited the participant sample to highly educated individuals, who were moreover relatively technologically savvy and open to adopting new technologies. However, a number of measures were taken to preserve validity of the findings to the degree possible. The volunteers were selected from a relatively large pool of over 300 employees, including students and administrative personnel. None of the participants had any prior knowledge of or experience with the related research. Any employees in the organization working for the same department as the members of the research team were excluded from the participants' pool. The research team kept each participant's identification anonymous from all the others in the organization.

\subsection{Procedure}

The study consisted of 3 phases, as illustrated in Fig. 3.

Pre-intervention (4 weeks): During the pre-intervention phase, the participants were given a pedometer. The researchers suggested that participants wear their pedometers as much as possible but did not set any goals, and encouraged participants to maintain their regular lifestyles. At the end of this phase, the cumulative reading of each pedometer was used to establish individual baselines, and to set goals for the study's second phase. 
Intervention (6 weeks): During the second, experimental phase, the participants were randomly assigned to one of the two experimental conditions. During this phase, the participants were encouraged to increase their daily step count to achieve their individual goals either during their work hours or during their leisure time. The participants were asked to make a daily stop at the public kiosk to update their pedometer number. Otherwise, the participants were free to engage with the game as little or as much as they wished; no additional interaction was required.

Post-intervention (4 weeks): At the end of the 6 weeks of the intervention phase, the game ended. However, participants were encouraged to continue wearing their pedometers for an additional 4 weeks. The final number captured by the pedometer at the end of the post-study phase allowed the researchers to observe any persistent effects of the game.

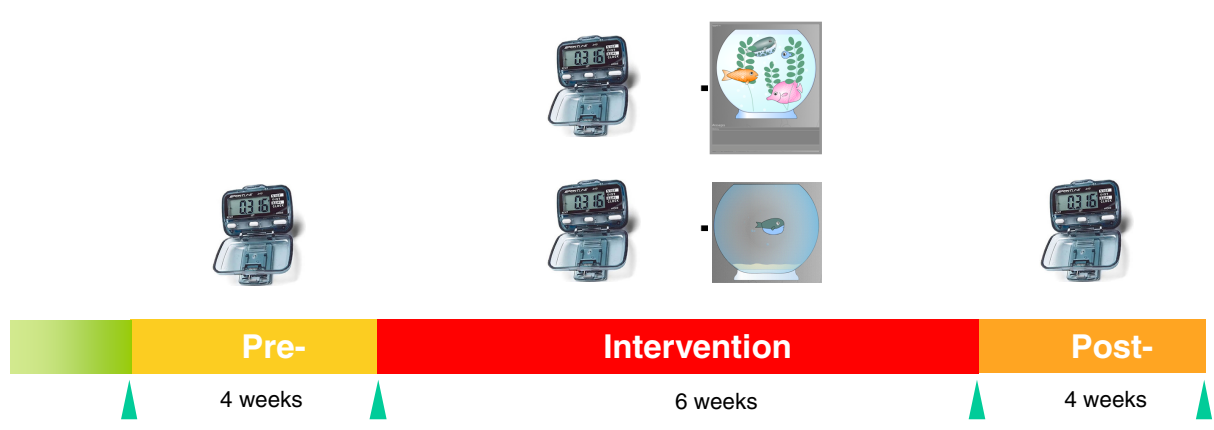

Fig. 3. Study overview. Qualitative interviews (4 total, indicated by triangles) were conducted between each of the phases in the study.

The mix-design of the study in two experimental conditions (team condition vs. single condition) allowed separate assessment of emotional attachment to the virtual pet, and the second condition that combined attachment to the virtual pet with social dynamics.

At the beginning and at the end of each intervention phase, each participant was interviewed for about 30 minutes to discuss their experiences. Researchers used handwritten notes to summarize interviews 1 and 2. Interviews 3 and 4 were video and audio-taped, and then transcribed. All the interview transcripts were coded according to categories that emerged during the study's analysis. The results were analyzed based on the data collected from the 19 participants who completed the entire 14week study. Among these 19 participants, 6 were in the single-player condition and 13 were in the multi-player condition.

The research team chose not to use a control group who wore pedometers but had no other incentives. Many previous pedometer studies, including Tudor-Locke [5] and Chan [15] , reported a plateau in their participants' daily step count after the studies' first 4 weeks of pedometer use. Following this pedometer "adoption" phase, the participants did not increase their daily step totals. For this study, we expected that 
effects due to the pedometers themselves would have been demonstrated in the first 4week pre-study phase. Therefore, increases in daily step counts observed during the game were likely reflecting the additional incentives introduced by the game.

\section{Findings}

To assess the achieved advancement along the steps of behavior change suggested by the Transtheoretical Model, the research team applied the following methodology. Each pre-study interview was analyzed for evidence of the participants' current position within the stages of the Transtheoretical Model: 1) Pre-contemplation - individuals have no recognition of the need to change and, consequently, no intention to take action; 2) Contemplation - intention to take action within foreseeable future (next six months); 3) Preparation - intention to take action within immediate future (next 30 days and having taken initial preparatory steps); 4) Action - practicing new behavior for 3 to 6 months; 5) Maintenance - continuing commitment to sustaining behavior; 6) Termination - overt behavior will never return, and there is complete confidence that one can cope without fear of relapse. For exercise programs, termination means that the behavior is so ingrained that external reinforcements are no longer necessary.

Similar analysis was applied to the post-intervention interviews, in order to determine whether there were indications of progression towards more advanced stages of behavior change. The number of steps recorded by the pedometer was the primary indicator of each participant's behavior change. In addition, the interviews provided another way to measure attitude changes.

To complement these quantitative findings, grounded theory analysis was used to identify recurrent patterns of attitudes towards the game through analysis of each round of interviews. All the interview transcripts were coded according to categories that emerged during the analysis.

\subsection{Initial Attitudes - Fish Buzz}

The rumors about the game started spreading through the building several months prior to the study, and led to a general feeling of anticipation and excitement. Pictures of the study's fish, while still a work in progress, were shared among the extended intern community at Siemens Corporate Research, and were observed throughout the building - attached to name tags, next to office cubicles, or attached to windows.

Not surprisingly, many of the recruited participants had noticed the pictures of the fish and were intrigued by them. In addition, many of the participants had been dissatisfied with their own level of physical activity, and welcomed an opportunity to address the issue. This was not surprising given the general work style at Siemens Corporate Research: sitting in front of one's computer all day long, as well as the "drive everywhere" culture of suburban living for many participants. Thus, the average level of daily steps determined during the pre-study phase was under 7,000 - quite a bit lower than the recommended 10,000 steps per day. 


\subsection{Observable Behavior Change}

According to the level definitions in the TTM, the following criteria were used to place participants in particular levels:

Table 1. TTM assessment results - pre-study

\begin{tabular}{|c|c|c|}
\hline Level & Definition & Example evidence \\
\hline 1 & $\begin{array}{l}\text { No intention to take action in the } \\
\text { next } 6 \text { months }\end{array}$ & $\begin{array}{l}\text { "I had a membership at a gym last year, but } \\
\text { dropped out. I don't have time anymore because } \\
\text { of the baby." } \\
\text { "I don't have an exercise routine or goal. I am } \\
\text { not member of a gym. Doctor suggested me to } \\
\text { do more exercising, but I didn't follow that } \\
\text { advice." }\end{array}$ \\
\hline 2 & $\begin{array}{l}\text { Intention to take action within the } \\
\text { next } 6 \text { months }\end{array}$ & $\begin{array}{l}\text { "I am member of a gym, but I have never been } \\
\text { there. I am thinking about going there." }\end{array}$ \\
\hline 3 & $\begin{array}{l}\text { Intention to take action within the } \\
\text { next } 30 \text { days and some initial } \\
\text { steps towards that action }\end{array}$ & $\begin{array}{l}\text { "I don't have an exercise routine. But I want to } \\
\text { develop one now and hope that this study helps } \\
\text { me. I have never been in a gym. My exercise } \\
\text { goal is to start exercising now." }\end{array}$ \\
\hline $4-5$ & $\begin{array}{l}\text { Change in behavior occurred at } \\
\text { some point in the past, but have } \\
\text { not developed into habit }\end{array}$ & $\begin{array}{l}\text { "I have a routine: } 3 \text { days a week squash and } \\
\text { weights, } 45 \text { min total. I am member of a gym } \\
\text { and usually exercise there." }\end{array}$ \\
\hline 6 & $\begin{array}{l}\text { Overt behavior will never return, } \\
\text { and there is complete confidence } \\
\text { in coping without tear of relapse. }\end{array}$ & $\begin{array}{l}\text { "I have had the same morning routine for } 11 \\
\text { years now; it includes a jog and an hour of yoga } \\
\text { and then my coffee and a newspaper" }\end{array}$ \\
\hline
\end{tabular}

As a result of this assessment, the following pattern emerged:

- Level 1: 4 participants. These individuals had not established exercise routines, nor had any intension of establishing them in a foreseeable future.

- Level 2: 1 participant. This individual had no established routine, but had recognized this as a limitation and was developing plans for changing the situation. However, no action has been taken to realize the plans.

- Level 3: 6 participants. Individuals in this category were actively forming exercise plans when they joined the game. Some of them have joined gyms, but have not started exercising yet, or had previously attempted exercise but did not succeed at sustaining it.

- Level 4-5: 4 participants. Individuals in this category had an established exercise routine, but they did not form a habit yet. Because it was not always possible to determine the exact time when the routine was formed (less than 6 months ago or over 6 months ago), we have combined levels 4 and 5 into one level.

- Level 6: 4 participants. These individuals have followed established and rigorous exercise routines for many years and had indicated strong habits.

A similar analysis of post-game interviews, including interviews 3 and 4, produced the following results (Table 2): 
Table 2. Cumulative TTM assessment results - pre-study and post-study. Gray rows indicate participants who achieved positive change in their daily steps.

\begin{tabular}{r|r|r|r|r|r}
\hline$\#$ & Pre-study level & Pre-study steps & Post-study level & Post-study steps & Change \\
\hline 1 & 1 & 5,175 & 4 & 7,000 & 1,825 \\
\hline 2 & 1 & 6,214 & 4 & 4,572 & $-1,642$ \\
\hline 3 & 1 & 7,140 & 2 & 6,676 & -464 \\
\hline 4 & 1 & 5,880 & 2 & 1,800 & $-4,080$ \\
\hline 5 & 2 & 4,904 & 2 & 3,742 & $-1,162$ \\
\hline 6 & 3 & 3,868 & 4 & 7,596 & 3,728 \\
\hline 7 & 3 & 7,610 & 3 & 6,880 & -730 \\
\hline 8 & 3 & 5,849 & 4 & 8,832 & 2,983 \\
\hline 9 & 3 & 1,884 & 3 & 1,920 & 36 \\
\hline 10 & 3 & 5,104 & 4 & 9,494 & 4,390 \\
\hline 11 & 3 & 6,546 & 4 & 11,725 & 5,179 \\
\hline 12 & $4-5$ & 3,705 & $4-5$ & 6,016 & 2,311 \\
\hline 13 & $4-5$ & 7,756 & 5 & 10,616 & 2,860 \\
\hline 14 & $4-5$ & 11,667 & 5 & 15,012 & 3,345 \\
\hline 15 & $4-5$ & 6,666 & $4-5$ & 7,026 & 360 \\
\hline 16 & 6 & 9,378 & 6 & 9,187 & -191 \\
\hline 17 & 6 & 10,284 & 6 & 16,496 & 6,212 \\
\hline 18 & 6 & 11,639 & 6 & 10,021 & $-1,618$ \\
\hline 19 & 6 & 7,171 & 6 & 8,123 & 952 \\
\hline
\end{tabular}

Level 1, Pre-contemplation: All four individuals in Level 1 clearly indicated increased awareness of the low levels of their activity:

"It made me conscious, conscious that I need to exercise more..."

At the same time, this newly gained awareness rarely led to any significant change in either their exercise routines, or the level of daily steps. In fact, for most of them the number dropped below the pre-study baseline:

"The game and fish made me realize that I am walking so little. It made me conscious. But I was very busy and didn't have time for exercising. The consciousness overall increased. But I didn't change my exercise goals or routines. I will continue wearing the pedometer. I think if I start with the game now again, it would have impact."

Two of the participants in this group took immediate action; however, only one of their reports was supported by an actual increase in the number of steps (from 5,000 to 7,000 daily):

"It motivated me to start exercising. Before I wasn't doing any kind of jogging. The game made me realize that I need to exercise more. My goals have now increased. I try to jog now on a daily basis for 5-10 min.. I am planning to do more; I try to walk and exercise more in general."

Level 2, Contemplation: The only participant in this category did not demonstrate any engagement in the game, found wearing the pedometer inconvenient and uncomfortable and participating in the study unjustifiably effortful. This participant did not 
demonstrate any increase in the number of daily steps; on the contrary, the number decreased by over 1000 steps:

"It was too much effort to log in each day and I didn't have information about my team members. I didn't like to wear the pedometer. It's inconvenient."

Level 3, Preparation: The six participants in this category were already actively preparing for increasing their physical activity. For three of them, the game seems to have provided just the kind of additional motivation that was wanted. For another participant, the game coincided with the diagnosis of asthma and a doctor's recommendation to increase daily exercise. All of these participants increased the number of steps to varying degrees (from 36 steps to over 5,000 steps daily). Even more importantly, many of them indicated their commitment to sustaining their increases even after the game:

"The game had impact on my activity. I did more walking outside and inside; went on the treadmill - to walk away my pounds. I curtailed my appetite at lunch; that was good. I am walking everyday now; this game gave me more incentive to walk more each day, because I wanted to be competitive. I walked for about 45 minutes each day. I am at the point now where I will just walk anyways; I don't need the contest anymore. It motivated me to continue with this speed."

The two participants who did not demonstrate improvement attributed it to unusually heavy workload throughout the game:

"It makes me aware of exercising. I am very busy right now because of [heavy workload], so I didn't spend much time for exercising. It had impact on the weekends: I tried to walk around more, do a few more exercise, or take the stairs instead of the elevator. It helped me to stay interested in activity."

Level 4-5, Action and maintenance: As was expected, individuals on higher levels of TTM, who already took an action to establish an exercise routine, did not report significant changes in their exercise habits. However, even for these individuals the game presented a new concrete challenge and a target to strive for: a number of daily steps. All participants in this category increased their steps by 2,000 - 3,000 steps a day:

"I got up more in the evenings. I tried to reserve time from 8pm to 10pm for exercising. I developed a concrete goal: at least 10,000 each day, but that was very ambitious. So I made the goal smaller and could keep it every day then. Part of the goal was to increase speed."

Level 6, Termination: Similar to the Level 4-5 participants, the changes in attitudes were not as drastic as for those on the lower levels. All of these participants continued to maintain their well-established exercise routines; however, several of them became more mindful about their walking habits:

"I was already very active before. I enjoyed the graphical representation about my activity level. In addition to my exercise routine I walked more in the office and parked far away or used the stairs instead of the elevator." 
In summary, application of the Transtheoretical Model for assessment of behavior change achieved in the course of the study demonstrated that Fish'n'Steps had a positive impact on 14 out of 19 participants as evident in either increase of their daily steps (for 4 participants), or advancements in TTM levels (for 3 participants) or both (for 7 participants).

The pattern of change across different levels very clearly indicated that individuals on the lowest (pre-contemplation) and highest (termination) levels were the least likely to achieve a significant change in their daily walking routines. The model provides a convincing explanation for this pattern: individuals in the pre-contemplation phase need to achieve a significant shift in attitude before demonstrating any observable change in behavior. At the same time, for individuals with well-defined exercise routines, the game did not generate any new awareness or motivation; these individuals could engage with the game for fun, but did not have a need to draw incentives from it.

Difference between conditions. Application of TTM analysis demonstrated no apparent differences in the achieved behavior change between the two experimental conditions as reflected by either the increase in the number of steps, or in the advancement in the TTM levels (see Table 3).

Table 3. Difference in achieved behavior change between the two experimental conditions

\begin{tabular}{l|r|r}
\hline Type of improvement & Group condition & Individual condition \\
\hline Average advancement in TTM levels & 1,076 & 1 \\
\hline Average increase in number of steps & 1,186 & 1,478 \\
\hline
\end{tabular}

\subsection{Reflecting on Intervention Design}

In general, the game served its purpose: it created initial excitement, increased participants' awareness of their levels of physical activity and it provided motivation to increase their activity in a fun and engaging way. At the same time, the study allowed the research team to reflect on the design of this type of intervention.

\section{Positive and negative incentives}

A significant number of study participants (14 out of 19) developed a certain emotional attachment to their virtual pet: they mentioned feelings of guilt or happiness associated with their fish being sad or happy.

"I was really happy when I saw my fish grow. I tried to motivate myself to walk more to make it grow. I have even increased my regular walking activities; when I saw that the fish had grown I felt very happy."

"The fish is part of me now. It was very good to have it as a motivation in the beginning. I miss the fish now."

Most of these participants realized and appreciated the little extra motivation the fish was providing:

"It is much more friendly than an excel spreadsheet, it would help keep an interest in exercising because one of the problems of exercises it to be a boring routine." 
Unfortunately, this emotional attachment could at times backfire: when their fish did not grow, or had sad emotions, some of the participants chose to not look at their fish at all, rather than watch their unhappy fish.

\section{"I didn't want to check on it, because I knew it was going to be sad."}

Based on the classification of the participants according the levels of the Transtheoretical Model, the participants on both, the lowest and the highest levels were less likely to engage with the game and develop a sense of attachment to their virtual pet. Those on the lowest levels often were often discouraged by the overly negative state of their fish; those on the highest levels had no perceived need for additional motivation. However, participants classified on levels 3 to 5 demonstrated the highest general level of engagement with the game and with their virtual characters.

\section{Cooperation and competition}

The two experimental conditions were created to assess separately the effect of the virtual character, the fish, and the effect of the engagement in a social game. The application provided a number of mechanisms to facilitate such social engagement: participants were split into teams of four and provided with access to each others' progress and opportunity to communicate via chat. In addition, both individual and public displays highlighted the team with the highest daily achievement to facilitate bonding between members of each team and promote team spirit.

The competitive aspect of the game elicited mixed reactions in the participants. For some of them competitiveness was a more enduring motivation than the virtual character:

"It was cool for the first one or two weeks, but then as you are in the first place you think the fish can jump out of the bowl and I don't care; the fact that I was in the first place it's all I need; it means that I was competitive so the fish didn't have much of an effect."

At the same time, some felt competitiveness to be unnecessary and incompatible with the spirit of the game:

"There is enough competition in real life; I didn't really need more."

However, for a majority of the participants, having other fish presented a stimulating challenge and a benchmarking mechanism. Many of them compared their own virtual character's states, moods and sizes to those of their team members and were aware of their comparative performance.

"I was wondering who the other people behind the fishes were, the other fishes in my team were always crying, so I was wondering if my team members were working on it.", “...one fish in my team was really small the whole time, the others also got pretty big in the end. I was wondering, why the person with the small fish was not working."

At the same time, as discussed above, application of TTM to the two conditions separately did not demonstrate any difference in achievement between the two conditions. 
In addition, providing mechanisms for anonymous team cooperation did not produce significant results. The majority of the participants felt awkward about contacting their team members without knowing who they were. The few that tried (3 out of 19) were quickly discouraged by the lack of response. Instead, because all of the participants were physically collocated and many knew each other well in person, the game was discussed actively over lunches, during coffee breaks, or other informal moments of face-to-face contact. On occasion, and especially at the beginning of the game, the participants were logging on to the same PC to compare their characters' moods and sizes.

\section{Sustainability of the intervention}

Despite the initial excitement about the game, the virtual characters and team competition, the game was perceived as increasingly repetitive as the study continued. While many of the participants (10 of 19) continued to have daily interactions, others limited their interaction to a few times a week. In addition, the inexpensive pedometers used for the study were meant to be worn on a belt, which was inconvenient for the female participants. All these aspects challenged continuous sustainability of the game.

"It was exciting at the beginning but then it turned into a nuisance; you would forget to put it on, or you would forget to log it in and stuff like that... I have to remember a lot of things during the day and that is on top of that..."

However, the game was not intended as a sustainable intervention, but as a temporary means of assisting the participants in advancing along the steps of behavioral change as proposed in the TTM.

\section{Conclusions}

Fish'n'Steps application was designed to provide multiple levels of incentives for increase in physical activity, which could be measured by a pedometer. These incentives included, on an individual level, the growth and emotional state of the individuals' virtual pets - fish in a fish-tank. Additional motivation was provided for participants in a team condition and included competition between teams with announcements of winning teams, and comparison between the states of the fish belonging to different members of the same team. To assess the potential of Fish'n'Steps to promote increase in individuals' activity levels, a 14-week study included 4 weeks of pre-study phase, in which individuals wore pedometers to establish their daily averages as a baseline; 6 weeks of participation in the game; and 4 weeks of post-study phase in which the game was terminated, but individuals continued to wear pedometers to assess sustainability of the potential change in their activity levels. As a result, 14 out of the 19 participants who completed the study demonstrated either advancement in the levels of Transtheoretical Model, or increase in the number of daily steps, or, and most commonly, both.

In general, the game seems to have served its purpose: created initial excitement, increased participants' awareness of their levels of physical activity and provided motivation to increase the activity level in a fun and engaging way. Although the initial fascination with the game subsided after the first couple of weeks, it nonetheless generated sustainable change in behavior and made continuing the game unnecessary. 
It also became evident that participants' ability to take advantage of the game depended to a large degree on their current level of physical activity, their satisfaction with it, their desire to change it, and consequently their position within the TTM levels. Participants in pre-contemplation level (1) were most likely to change their attitude, but less likely to produce visible increase in the number of steps, as compared to other levels. Participants in termination level (6) were less likely to change either their attitude or the number of steps, as compared to other levels. And participants in the intermediate levels, preparation, action and maintenance, were most likely to change both, their attitude and their actual behavior.

The study also allowed researchers to reflect upon the design of the interventions. For example, the virtual pet character was designed to provide positive reinforcement to the desired behavior (happy growing fish) and negative reinforcement to the lack of the desired behavior (crying, not growing fish). While many participants felt certain level of responsibility for their character and tried to prevent it from crying, some reduced interactions with the game to avoid encounters with a crying fish.

To promote cooperation between the team members in a team condition, the application provided a possibility of chat communication. In addition, in order to preserve participants' privacy, they were encouraged to pick screen names. This combination created a surprising effect: instead of using chat for communication, participants tried to find each other and discuss the game during lunches, coffee breaks, or other informal face-to-face encounters. Many participants felt awkward contacting their team members without knowing who they really were. This approach of anonymous virtual communication might be more appropriate for distributed community in which members do not know each other.

One of the limitations of the game that prevented participants from engaging with it was intrusiveness of devices and procedures. The participants patiently followed the procedures of the study to assist researchers, however, most commented on the inconvenience of wearing the pedometer and uploading the data in the public kiosk. Less obtrusive uploading mechanisms might contribute to sustainability of the game.

\section{References}

[1] Orzano, A. J., Scott, J. G. (2004). Diagnosis and Treatment of Obesity in Adults: An Applied Evidence-Based Review. J Am Board Fam Med 17: 359-369.

[2] Purnell, J.Q., Knopp, R.H., Brunzell, J.D., Diatary fat and obesity, American Journal of Clinical Nutrition, 1999, pp.70-108.

[3] Bacon, L., Keim, N.L., Van Loan, M.D., Derricte, M., Gale, B., Kazaks, A., Stern, J.S., Evaluating a 'non-diet' wellness intervention for improvement of metabolic fitness, psychological well-being and eating and activity behaviors, International Journal of Obesity (2002) 26, 854-865.

[4] Bissell, P., Compliance, Concordance and Respect for the Patient's Agenda, The Pharmaceutical Journal, vol. 271, October 2003, pp.498-500.

[5] Tudor-Locke, C.E., Myers, A.M., Bell, R.C., Harris, S.B., Rodger, N.W., Preliminary Outcome Evaluation of the First Step Program: a daily physical activity intervention for individuals with type 2 diabetes, Patient Education and Counseling, 47 (2002) pp.23-28. 
[6] Grimley, D., Prochaska, J.O., Velicer, W.F., Vlais, L.M., and DiClemente, C.C., 1994. The transtheoretical model of change. In T.M. Brinthaupt \& R.P. Lipka, Changing the self: Philosophies, techniques, and experiences. SUNY series, studying the self (p. $201-$ 227). Albany, NY: State University of New York Press.

[7] Bandura, A., 1982. Self-efficacy mechanism in human agency. American Psychologist, 37 (2), $122-147$.

[8] DiClemente, C.C., Prochaska, J.O., Fairhurst, S.K., Veliceer, W.F., Velasquez, M.M, and Rossi, J.S., 1991, The process of smoking cessation: An analysis of precontemplation, contemplation, and preparation stages of change. Journal of Consulting and Clinical Psychology, 59, 295 - 304.

[9] Dallow CB, Anderson J. (2003).Using self-efficacy and a transtheoretical model to develop a physical activity intervention for obese women. American Journal of Health Promotion, 17, 373- 81 .

[10] Marshall S., Biddle S. (2001). The transtheoretical model of behavior change: a metaanalysis of applications to physical activity and exercise. Annals of Behavioral Medicine, 23, 229- 46.

[11] Riebe D., Blissmer B., Greene G., Caldwell, M., Ruggiero, L., Stillwell, K., Nigg, C. (2005). Long-term maintenance of exercise and healthy eating behaviors in overweight adults, Preventive Medicine, 40, 769-778.

[12] Lieberman, D.: Dance Dance Revolution: The Most Researched Serious Game Ever. Why, and What Have We Learned? https://www.cmpevents.com/GDsg05/a.asp?option=C\&V=11\&SessID=1075, Serious Games Summit DC 2005 Sessions.

[13] Mokka, S., Väätänen, A., Heinilä, J., Välkkynen, P., Fitness computer game with a bodily user interface, Proc ICEC '03, pp.1-3.

[14] Björk, St., Holopainen, J., Ljungstrand, P., Akesson, K.P., Designing Ubiquitous Computing Games - A Report from a Workshop Exploring Ubiquitous Computing Entertainment, Personal and Ubiquitous Computing (2002), pp.443-458.

[15] Chan, C., Ryan, D., Tudor-Locke C. (2004). Health benefits of a pedometer-based physical activity intervention in sedentary workers, Preventive Medicine, 39, 1215-1222.

[16] Consolvo, S., Everitt, K., Smith, I., Landay, J.A., Design requirements for technologies that encourage physical activity. Proceedings of the SIGCHI conference on Human Factors in computing systems, 2006, pp.457-466.

[17] Hardeman, W., Griffin, S., Johnston, M., Kinmonth, A.L., Wareham., N.J., Interventions to prevent weight gain: a systematic review of psychological models and behaviour change methods, International Journal of Obesity (2000) 24, 131-143.

[18] Langer, E.J., Rodin, J., 1976, The Effects of Choice and Enhanced Personal Responsibility for the Aged: A Field Experiment in an Institutional Setting, Journal of Personality and Social Psychology 34: 191-198.

[19] Lieberman, D., Interactive Video Games for Health Promotion: Effects on Knowledge, Self-Efficacy, Social Support, and Health, Health Promotion and Interactive Technology: Theoretical Applications and Future Directions, 1997, Mahwah, NJ: Lawrence Erlbaum Associates.

[20] McLean, N., Griffin S., Toney, K., and Hardeman, W., Family involvement in weight control, weight maintenance and weight-loss interventions: a systematic review of randomized trials, International Journal of Obesity (2003) 27, 987-1005.

[21] Intille, S. S. (2003). Ubiquitous Computing Technology for Just-in-Time Motivation of Behavior Change (Position Paper), in Proceedings of the UbiHealth Workshop' 2003. 
[22] Jafarinaimi, N., Forlizzi, J., Hurst, A., and Zimmerman, J. (2005). Breakaway: an ambient display designed to change human behavior. In CHI '05 Extended Abstracts on $\mathrm{Hu}-$ man Factors in Computing Systems (Portland, OR, USA, April 02 - 07, 2005), 19451948.

[23] Fogg, B.J., Persuasive Technology : Using Computers to Change What We Think and Do, Morgan Kaufmann (December 2002).

[24] Konkel, M., Leung, V., Ullmer, B., Hu, C., Tagaboo. (2004). A collaborative children's game based upon wearable RFID technology, Pers Ubiquit Comput, pp.382-384.

[25] Croteau, K. (2004). A preliminary study on the impact of a pedometer-based intervention on daily steps, American Journal of Health Promotion, 18, 217-220. 